\section{SLE: EULAR/ACR-Klassifi- kation 2019 auch bei Subgruppen sehr sensitiv
und spezifisch}

Johnson SR et al. Performance of the 2019 EULAR/ACR classification criteria for systemic lupus erythematosus in early disease, across sexes and ethnicities. Ann Rheum Dis 2020; 79 : 1333-1339

Die Klassifikationskriterien für systemischen Lupus erythematodes (SLE) der European League Against Rheumatism (EULAR) und des American College of Rheumatology (ACR) von 2019 zeigten in der Validierungsstudie eine hohe Sensitivität und Spezifität. In einer neuen Studie wurde untersucht, wie sensitiv und spezifisch die Kriterien bei der Diagnosestellung in bestimmten Subgruppen (frühe Erkrankung, Männer, bestimmte Ethnien) sind.

Um die neuen Kriterien validieren zu können, riefen die Wissenschaftler Ärzte aus 21 SLE-Zentren aus 16 Ländern (Österreich, Kanada, Kroatien, Frankreich, Deutschland, Griechenland, Hong Kong, Ungarn, Japan,
Italien, Mexiko, Portugal, Spanien, Türkei, Vereintes Königreich, USA) dazu auf, ihnen Fälle von SLE-Patienten und passenden Kontrollen zu schicken.

Die so entstandene Validierungskohorte $(n=1270)$ bestand aus 1098 weiblichen, 172 männlichen, 118 asiatischen, 68 schwarzen, 124 hispanischen und 941 weißen $\mathrm{Pa}$ tienten. Bei 34 Patienten betrug die Krankheitsdauer unter einem Jahr, bei $196 \mathrm{~Pa}$ tienten zwischen einem bis $<3$ Jahren und bei $879 \geq 5$ Jahren.

Die Autoren verglichen die Sensitivität und Spezifität der neuen EULAR/ACR-Kriterien außerdem mit den Systemic Lupus International Collaborating Clinics (SLICC) 2012 und ACR 1982/1997 Kriterien.

Die Auswertung erbrachte u. a. folgende Ergebnisse:

- Die neuen EULAR/ACR-Kriterien zeigten insgesamt hohe Sensitivitäten und Spezifitäten, bei Männern 93\% Sensitivität und $96 \%$ Spezifität, bei Frauen $97 \%$ Sensitivität und $94 \%$ Spezifität.

- Bei Patienten mit einer Krankheitsdauer von 1 bis < 3 Jahren ergaben die neuen EULAR/ACR-Kriterien eine Spezifität von $96 \%$ und Sensitivität von $97 \%$.

- Bei Patienten mit einer Krankheitsdauer von 1 bis < 3 Jahren ergaben die neuen EULAR/ACR-Kriterien eine höhere Sensitivität als die ACR-Kriterien (97 vs. $81 \%$ ).

- Bei Frauen ergaben die neuen EULAR/ ACR-Kriterien eine höhere Sensitivität als die ACR-Kriterien (97 vs. $83 \%$ ) und eine höhere Spezifität als die SLICCKriterien (94 vs. $82 \%$ ).

- Bei weißen Patienten hatten die EULAR/ACR-Kriterien eine höhere Sensitivität als die ACR-Kriterien (95 vs. $83 \%$ ) und eine höhere Spezifität als die SLICC-Kriterien (94 vs. $83 \%$ ).

- Die EULAR/ACR-Kriterien schnitten bei schwarzen Patienten gut ab (Sensitivität von $98 \%$, Spezifität $100 \%$ ) und hatten eine höhere Sensitivität als die ACRKriterien bei hispanischen Patienten ( 100 vs. $86 \%$ ) und asiatischen Patienten (97 vs. $77 \%$ ). 
FAZIT

Die vorliegende Studie zeigt, dass die EULAR/ACR 2019 Kriterien zur Diagnose des systemischen Lupus erythematodes auch bei Patienten mit früher Erkrankung, Männern und Patienten bestimmter Ethnien sehr spezifisch und sensitiv sind. In bestimmten Subgruppen haben sie höhere Sensitivität und Sensitivität als die ACR 1982/1997 und SLICC 2012-Kriterien.

Marisa Kurz M. Sc. B. A. München 